(2) The Editor of the Referativny Zurnal, Astronomija i Geodesia, Moskva D-2I9 Baltijskij Pos. D. 42B.

(3) L'éditeur du Bulletin Analytique du C.N.R.S., 45 rue d'Ulm, Paris-V, France.

(4) L'éditeur des Astronomical News Letters, Laboratoire d'astronomie, Porte de Douai, Lille (Nord), France.

2. La Commission 5 prie l'U.A.I. de prévoir pour la prochaine période de trois ans, annuellement, une somme de I500 dollars (crédit total 4500 dollars) de manière à assurer la continuation de la publication des Astronomical News Letters sous la direction du Dr V. Kourganoff du Laboratoire d'astronomie de l'Université de Lille. Il est entendu que le Dr Kourganoff, d'accord avec le président de la Commission 5, préparera une liste des instituts astronomiques qui recevront gratuitement les A.N.L. Le nombre total des instituts qui recevront de cette manière les $A . N . L$. ne dépassera pas, dans le cadre du crédit prévu ci-dessus, deux cents.

3. La Commission témoigne sa satisfaction quant à l'état d'avancement du travail entrepris pour combler la lacune entre la Bibliographie générale de l'astronomie de Houzeau et Lancaster et l'Astronomischer Jahresbericht, et insiste pourque les dispositions nécessaires soient prises pour terminer le manuscrit avant la prochaine Assemblée générale de l'U.A.I.

4. La Commission, appréciant l'étendue de la diffusion donnée aux Extraits d'astronomie $d u$ Bulletin analytique du C.N.R.S., estime que la subvention qui a été donnée à celui-ci doit être renouvelée à concurrence de 1500 francs-or pour la période des trois années à venir (1956-57-58).

5. La Commission, ayant pris connaissance de l'édition provisoire du Répertoire: Les observatoires astronomiques et les astronomes, et heureuse des résultats atteints, estime qu'il y aura lieu d'attribuer à l'Observatoire royal de Belgique une seconde et dernière subvention de 500 dollars pour permettre d'entreprendre l'édition définitive de cet ouvrage.

\title{
Commission 6
}

La Commission demande à l'U.A.I. de continuer sa subvention de I200 francs-or par an au Bureau Central des Télégrammes Astronomiques.

\section{Commission 8}

I. La Commission approuve unanimement les recommandations de la Conférence d'Astrométrie de Bruxelles du 28-30 mars I955. Ces recommandations ont été imprimées dans les Communications de l'Observatoire royal de Belgique, no. 85, pp. 37-42.

Les observatoires de Babelsberg et Ottawa ont été ajoutés à la liste primitive (p. 38) des observatoires qui prendront part aux observations méridiennes.

Le comité prévu au Nr. VII des Résolutions (p. 39) sera constitué par les spécialistes suivants: F. P. Scott (président), D. Brouwer, A. DAN Jon, W. Fricke, O. Heckmann, A. Nemiro, L. S. T. Symms, M. S. Zverev.

2. La Commission recommande que dans tous les catalogues méridiens, outre l'époque de l'observation pour chaque étoile soit donné au moins: le Temps Moyen d'Observation dans l'Année pour l'ensemble des observations de l'étoile; il serait préférable de donner les facteurs parallactiques en $a$ et $\delta$, si les dates des observations avaient une repartition défavorable.

3. L'Union Astronomique Internationale appelle l'attention sur la nécessité de nouvelles observations méridiennes dans l'hémisphère austral, et elle insiste auprès des gouvernements de l'Australie et de l'Afrique Australe pour que les crédits indispensables à la mise en service des instruments existants soient accordés.

\section{Commission 9}

Les convertisseurs d'images photoélectriques ont atteint un point de leur développement tel que leur supériorité sur les méthodes photographiques devient évidente en de 